\title{
Intercalibration experiment of methane standard gas scale between NOAA/CMDL and MRI/GRL
}

\author{
by \\ Hidekazu Matsueda \\ Meteorological Research Institute, Tsukuba, Ibaraki, 305 Japan \\ (Received February 21, 1993; Revised June 21, 1993)
}

\begin{abstract}
We developed an automated GC/FID analysis system to determine methane mixing ratios of standard gases with a mean precision of $\pm 0.12 \%$. The GC/FID analysis of five gravimetric standards showed that their methane scale was stable and internally consistent. However, the diluent air for these standards had a small amount of methane, which was estimated to be $35.5 \mathrm{ppb}$. Thus, our MRI/GRL scale was corrected for methane impurity in the diluent air.

The methane mixing ratio of dry natural air in an aluminum cylinder prepared for the intercalibration experiment was determined to be $1.7545 \pm 0.0015 \mathrm{ppm}$ on the basis of the MRI/GRL scale. This mixing ratio was higher by $23.0 \mathrm{ppb}$ than that assigned by NOAA/CMDL. This difference indicated that the offset of $23.0 \mathrm{ppb}$ between NOAA/ CMDL and MRI/GRL scales will need to be taken into accoount when methane data from both laboratories are compared.
\end{abstract}

\section{Introduction}

Since the late 1970's, accurate measurements of atmospheric methane have been made using gas chromatographs with flame ionization detectors (GC/FID). Atmospheric methane has been measured in air samples collected at many remote sites by the National Oceanic and Atmospheric Administration/Climate Monitoring and Diagnostics Laboratory (NOAA/CMDL) (Steele et al., 1987; Steele et al., 1992). Several other groups have also been measuring atmospheric methane according to their own sampling philosophies (Fraser et al., 1986; Khalil and Rasmussen, 1986; Blake and Rowland, 1988; Scheel et al., 1990; Aoki et al., 1992).

In our laboratory (Meteorological Research

C 1993 by the Meteorological Research Institute
Institute/Geochemical Research Laboratory, MRI/GRL), analysis of methane by GC-FID was first applied to maritime air samples collected from 1978 to 1986 (Matsueda et al., 1992). Since 1987, more frequent measurements have been obtained in clean Pacific air using research and commercial vessels (Matsueda et al., in preparation), and aircraft (Inoue et al., 1991; Matsueda et al., 1993). In addition, continuous measurements in surface air at Tsukuba city also started in 1988. Recently, measurements from the Pacific suggested that our methane standard gas scale was slightly different from that of NOAA/ CMDL (Matsueda et al., 1992), but the magnitude of the difference is unclear. It was necessary to intercompare methane standard gas scales to quantitatively determine this difference.

We use gravimetric methane-in-air stan- 
dards prepared by a gas company (Nippon Sanso Corporation, Japan: NSC), because we have no equipment for standard gas preparation in our laboratory. Recently, we found that a small amount of methane was contained in the diluent air used for preparation of NSC standard gases. This impurity is described in detail below. This indicates that methane mixing ratios assigned to standard gases are not correct in an absolute sense. Thus, an intercalibration experiment is important for our laboratory in evaluating the difference between an absolute standard scale and ours.

Methane intercalibration experiment between NOAA/CMDL and MRI/GRL was conducted on dry natural air in an aluminum cylinder (FF30507), which was prepared by NOAA/CMDL. The cylinder was sent to MRI/ GRL by Dr. E. J. Dlugokencky (NOAA/ CMDL) in April, 1992. The methane mixing ratio of the air in cylinder FF30507 was measured in MRI/GRL during May-June. In July, the cylinder was returned to NOAA/CMDL and then its methane mixing ratio was redetermined to make sure that it was not changed during the round trip. Finally, the result of this experiment was exchanged between NOAA/CMDL and MRI/GRL.

For this intercalibration experiment, we developed an automated analytical system for measurements of methane-in-air standards by GC/FID and then checked our methane standard gases. In the present study, we first describe our analytical procedure and our methane standard, and then report the results of the methane measurement for cylinder FF30507. On the basis of these results, we will discuss the difference of standard scale between NOAA/CMDL and MRI/GRL and adjustment factor of methane data previously reported by MRI/GRL against the NOAA/ CMDL calibration scale.

\section{Materials and method}

\subsection{Primary standard}

A gravimetric methane standard prepared by NSC was obtained for use as a primary stan dard in our laboratory. The methane mixing ratios of nine standard gases and their preparation dates are given in Table 1 . These standards are classified into three families (DJ, CPA and DF) according to the preparation date. The preparation method was the same for

Table 1 List of nine gravimetric methane standards and one diluent air.

\begin{tabular}{llll}
\hline Cylinder & Preparation & "Assigned" & "Corrected" \\
Number & date & $\mathrm{CH}_{4}$ mixing & $\mathrm{CH}_{4}$ mixing \\
& & ratio $^{*}(\mathrm{ppm})$ & $\mathrm{ratio}^{+}(\mathrm{ppm})$
\end{tabular}

\section{DJ Family}

DJ5459 July 14, $1988 \quad 1.7022$

DJ5460 July 14, $1988 \quad 1.8017$

\section{CPA Family}

CPA00179 July 25, $1989 \quad 1.6499$

CPA00538 July 25, $1989 \quad 1.7511$

\section{DF Family}

DF4728 April 1, $1991 \quad 1.5010 \quad 1.5365$

DF4727 April 1, $1991 \quad 1.6016 \quad 1.6371$

DF4797 April 1, $1991 \quad 1.7018 \quad 1.7373$

DF4723 April 2, $1991 \quad 1.8031 \quad 1.8386$

DF4737 April 2, $1991 \quad 1.9028 \quad 1.9383$

CPB00617 April 3, $1991 \quad 0.0000 \quad 0.0355$

*"Assigned" methane mixing ratio from gravimetric method was reported by NSC, but this value was calculated assuming no methane in the diluent air.

+"Corrected" methane mixing ratio was corrected for methane content in the diluent air (CPB 00617)

${ }^{8}$ CPB00617 cylinder was filled with the same diluent air as used for preparation of DF family. Estimation of methane content in this cylinder is described in the text.

the three groups. The basic procedure for gravimetric standard preparation by NSC is almost the same as those in Aoki and Kawaguchi (1990) and Aoki et al. (1992). The preparation procedure is briefly described below.

The gravimetric standards were made in high-pressure $10-\mathrm{L}$ aluminum cylinders fitted with brass or stainless steel valves. The stainless steel valves were used for five cylinders of the DF family. The inside wall surface of each cylinder was cleaned by steam and then dried with nitrogen gas. All the cylinders were treated by heating to $>100^{\circ} \mathrm{C}$ at a pressure of less 
than $5 \times 10^{-5}$ torr for more than 4 hrs. Prior to standard preparation, the cylinders were filled with methane in air (20ppm and 200ppm) to approximately $10 \mathrm{~kg} / \mathrm{cm}^{2}$ and maintained at room temperature for about 20 days. This preconditioning of the cylinders exposed the internal tank surface to air to minimize potential methane uptake or release from the tank surface. After the preconditioning, these cylinders were evacuated to the pressure of less than $5 \times 10^{-2}$ torr for the standard preparation.

High-purity methane ( $>99.999 \%)$ was used as a starting material for preparation of standard gases. This pure methane was gravimetrically diluted three times to prepare "parent" standard with relatively high concentrations $(30 \mathrm{ppm})$. The target range for methane, between $1.5 \mathrm{ppm}$ and $1.9 \mathrm{ppm}$, was prepared by gravimetric dilution of the "parent" standard using "ultrapure" air as a diluent gas. The preparation of five standard gases for the DF family required four diluent air cylinders filled with "ultrapure" air. In this study, about equal amounts of diluent air were used from four cylinders for the "parent" standard dilution to avoid the scatter of methane impurity. For the DF family, one of the $10-\mathrm{L}$ aluminum cylinders used for standard gas preparation was filled with diluent air only to determine the methane impurity.

"Assigned" methane mixing ratios of nine gravimetric standards reported by NSC are listed in Table 1, although these values were calculated assuming no methane impurity in the diluent air. The uncertainties on the "assigned" mixing ratios were $\pm 0.2 \%$. Most of the errors were associated with determining the change in weight of the cylinder. All mixing ratio units here are reported in parts per million (ppm) or parts per billion (ppb) by mole fraction in dry air.

\subsection{Secondary standard}

Several high-pressure cylinders filled with methane in air were used as secondary standards for measurements of atmospheric air samples. These standards were made in $10-\mathrm{L}$ or 47-L steel cylinders with brass fittings. The cleaning and preconditioning procedure of these cylinders was almost the same as that of the aluminum cylinder described above. The target range for methane, between 1.5 and 2.2 ppm, was obtained by volumetric methods used by NSC and TIC (Takachiho Chemical Industry Co. LTD., Japan). The standards from NSC and TIC were prepared using purified natural air and synthetic air as a diluent gas, respectively. The mixing ratios of these secondary gases were determined relative to the gravimetric standards.

\subsection{Analytical method}

Methane was measured with a gas chromatograph equipped with a flame ionization detector (Shimadzu GC-16A; Shimadzu Japan). Methane in air was separated by a stainless steel column ( $3 \mathrm{~mm}$ I.D. $\times 2 \mathrm{~m}$ long) packed with Molecular Sieve $13 \mathrm{X}-\mathrm{S}(60 / 80$ mesh) st $80^{\circ} \mathrm{C}$. Ultrahigh purity nitrogen ( $\left.>99.9999 \%\right)$ was used as a carrier gas at a flow rate of 50 $\mathrm{ml} / \mathrm{min}$. Ambient air used for the flame was pressurized by a compressor and then dried through a stainless steel cylinder packed with silica gel. After drying, this air was passed through a catalytic oxidation column to convert methane and non-methane hydrocarbons to $\mathrm{CO}_{2}$. Hydrogen was supplied to the FID by a hydrogen generator. The hydrogen generated was dried through a silica gel column and was then passed through a column packed with Molecular Sieve 5A to remove FID reactive gases. These cleaning procedures for air and hydrogen are necessary to obtain higher FID sensitivity and better stability of FID base line. Methane peak areas were measured using a chromatographic integrator (Chromatopac C -R4A; Shimadzu Japan), which stored raw chromatographic data to a 3.5 inch floppy disk.

Injection of standard air onto the GC column was performed using a six-port valve connected with a $5 \mathrm{ml}$ sample loop. The sample loop was kept in the GC oven to maintain a constant temperature. The sample loop was flushed by standard air for $1.5 \mathrm{~min}$ at a flow rate of $170 \mathrm{ml} / \mathrm{min}$, which was controlled by a mass flow controller. After flushing, the standard air flow was stopped by a two-posi- 
tion valve for $1 \mathrm{~min}$ to equilibrate the air in the sample loop to atmospheric pressure, prior to injection onto the GC column. A pressure gauge was used for monitoring atmospheric pressure to correct the small change in mass of the injection sample. This pressure data was stored in the chromatographic integrator ( $\mathrm{C}-\mathrm{R} 4 \mathrm{~A})$.

One of eight standard gases was selected using an eight-position stream selection valve. This valve was connected with the six-port valve to introduce the standard air from the pressurized cylinder into the sample loop. In some cases, a stainless steel tube immersed in an alcohol bath maintained at $-80^{\circ} \mathrm{C}$ between the six-port valve and the eight-position valve was used to insure a dry sample. The air supply for switching the valves was controlled by electric valves, which were operated through a relay interface (PRG-102A; Shimadzu, Japan) coupled to the chromatographic integrator (C-R4A). Thus, repetitive analysis of eight standard gases could be automated by the chromatographic integrator's basic program.

\section{Results and discussion}

\subsection{Primary standards}

Each analysis run consisted of 5 measurements so that the analytical variation of our GC/FID method could be estimated. We made 504 runs using the primary and secondary standards during June-July, 1991. Fig.1 shows the frequency distribution of the analytical reproducibility (1 standard deviation: 1 s.d.) for five repetitive measurements. All of the standard deviations were less than $0.3 \%$ and the mean value was $0.12 \%$. Although the mixing ratios of these standards ranged from 1.5 to 2.2 ppm, the analytical reproducibility was found to be independent of methane mixing ratio within this range.

The five gravimetric standards of the DF family were prepared by NSC to serve as the basis of a calibration scale for measurements of atmospheric methane. The internal consistency of these standards was evaluated by calibrating the methane mixing ratio of one cylinder using the other four cylinders as the

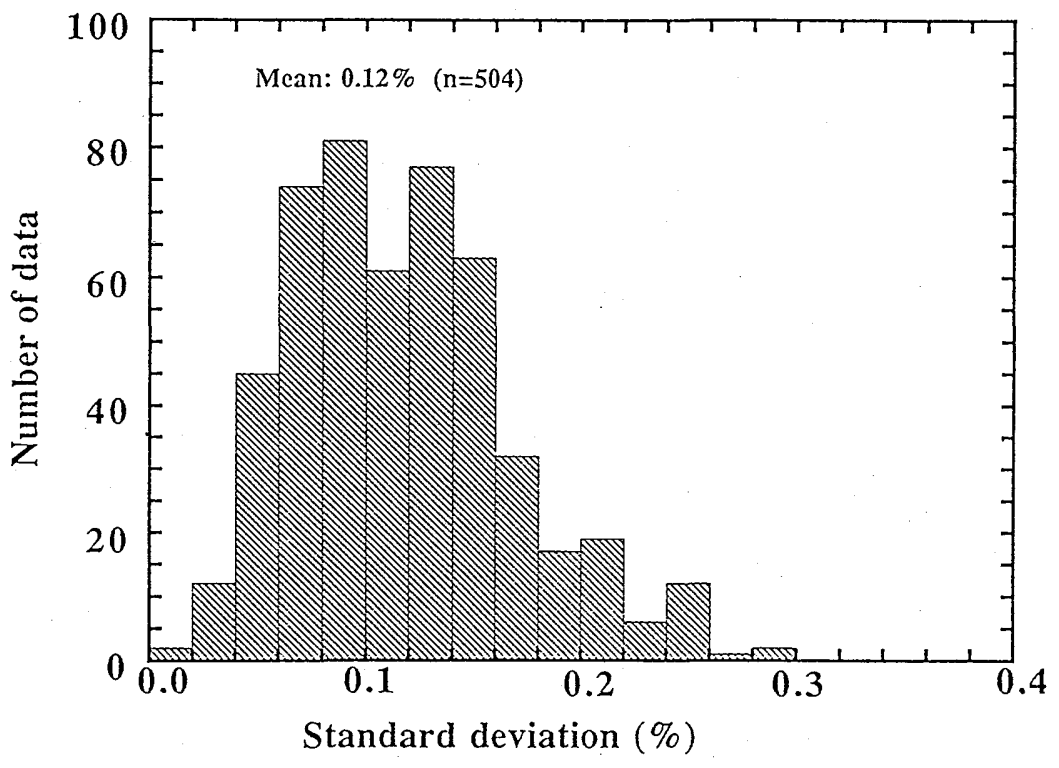

Fig. 1 Frequency distribution of standard deviations from methane analysis used in this work. Each standard deviation was calculated from 5 measurements. The total number of standard deviations is 504 , and its mean is $0.12 \%$. 
reference gases. The five gravimetric standards of the DF family were analyzed 75 times by GC/FID during June-July, 1991 to obtain a mean FID area response for each. Based on this result, the methane mixing ratio of each standard was calculated from the measured area response using the linear regression analysis on the measurements of the other four standards (Table 2). The measurements of four standards were highly linearly correlated $(r>0.999996)$ in all cases. The calculated values using the linear calibration lines agreed with the corresponding "assigned" values within $\pm 0.08 \%$. Considering the precisions of standard preparation and GC-FID analysis, the magnitude of these deviations was negligible. In this study, the linear regression analysis was sufficient for calculating methane mixing ratio within our analytical error. From these results, it can be seen that the NSC gravimetric methane scale is relatively consistent among the five standards of the DF family.

The gravimetric standards of DJ and CPA families were analyzed for methane during June-July, 1991 to compare them with the calibration line of DF standards. Fig.2 is a plot of mean FID response for 7 gravimetric stan dards (DF, DJ and CPA families) versus the "assigned" methane mixing ratio. We connected the 2 standards for families DJ and CPA by a straight line, to compare them with the linear regression line for the DF family.

A systematic difference was found smong the calibration lines for the DF, DJ and CPA families, although all standards were prepared by NSC according to the same gravimetric procedure. The three calibration lines have almost the same slope, but the $\mathrm{Y}$ intercepts of the straight lines were systematically different. It is probable that the disagreement among the calibration lines was caused by different amounts of methane in the diluent air used for the preparation of the three standard families. This discrepancy could not be demonstrated in this study, because we did not have the diluent air used to prepare the DJ and CPA standard families. However, the impurity of the diluent air in the DF standard family was confirmed by GC/FID analysis as described below.
Table 2 Comparison between "assigned" $\mathrm{CH}_{4}$ mixing ratio and calculated $\mathrm{CH}_{4}$ mixing ratio using measurements of DF standard family during June-July, 1991.

\begin{tabular}{lccc}
\hline $\begin{array}{l}\text { Cylinder } \\
\text { number }\end{array}$ & $\begin{array}{c}\text { "Assigned" } \\
\text { mixing ratio } \\
\text { (ppm) } \\
\text { (A) }\end{array}$ & $\begin{array}{c}\text { CalculatedCH} \\
\text { mixing ratio* } \\
\text { (ppm) }\end{array}$ & Percent \\
& residual & \\
\hline $\mathrm{DF} 4728$ & 1.5010 & 1.5022 & -0.080 \\
$\mathrm{DF} 4727$ & 1.6016 & 1.6009 & +0.044 \\
$\mathrm{DF} 4797$ & 1.7018 & 1.7016 & +0.012 \\
$\mathrm{DF} 4723$ & 1.8031 & 1.8028 & +0.017 \\
$\mathrm{DF} 4737$ & 1.9028 & 1.9038 & -0.053 \\
\hline
\end{tabular}

${ }^{*}$ Calculated $\mathrm{CH}_{4}$ mixing ratio was calculated from each measured area response using the best least squares fit to other four standard measurements.

${ }^{\S}$ Percent residual is defined as $[(\mathrm{A}-\mathrm{B}) / \mathrm{A}] \times 100$ and represents percentage deviation of the calculated $\mathrm{CH}_{4}$ mixing ratio to the "assigned" $\mathrm{CH}_{4}$ mixing ratio.

\subsection{Purity of diluent air}

The "ultrapure" air used as the diluent gas for the preparation of NSC standards was natural air purified according to a proprietary gas company procedure. The analytical report from NSC indicated a dew point of less than $-70^{\circ} \mathrm{C}$ for this "ultrapure" air. This report also suggested that trace amounts of hydrocarbons (methane) were contained in this diluent air, but the "assigned" methane mixing ratio was calculated assuming no methane in the diluent air.

In order to check the purity with respect to methane, we analyzed a cylinder (CPB00617) filled with the same "ultrapure" air used as the diluent for the preparation of five DF standards. Fig. 3 shows gas chromatograms of the diluent air (CPB00617) and ultrahigh purity nitrogen ( $>99.9999 \%$ ) compared with that of methane standard gas (DF4797). A small peak corresponding to trace amounts of methane was clearly observable in the chromatograms of the diluent air, but no methane was detected in the ultrahigh purity nitrogen with our GC/FID method. This result indicates that the methane mixing ratio "assigned" by NSC was not correct in an absolute sense due to the methane impurity in the diluent air.

An accurate determination of the low- 


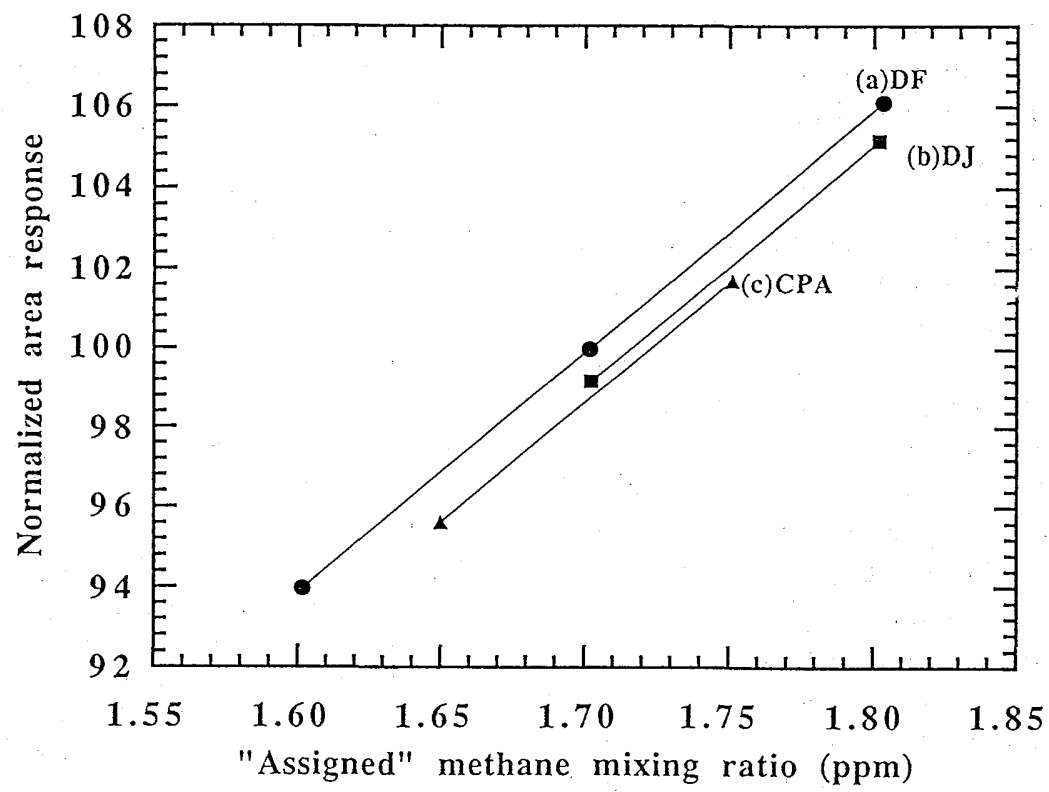

Fig. 2 Plot of the normalized area response of 7 gravimetric standards prepared by NSC versus the "assigned" methane mixing ratio. Each point is the mean of at least 35 measurements, normalized to the area response of the air in cylinder DF4797. The standard deviation of the replicate analyses is contained within the point. The three lines represent the least squares linear fits, one to each standard family.

level methane in the diluent air (CPB00617) was required to correct the "assigned" methane value, but a low-level methane standard was not prepared for the present study. Therefore, a preliminary estimation of the methane content in the diluent air was performed according to the following procedure. As shown in Fig.3, the slopes of the three calibration lines were almost identical, indicating constant sensitivity of the GC/FID to methane mixing ratio. Based on the average sensitivity of the FID methane response obtained during analysis of cylinder CPB00617, the methane content of the diluent air was calculated to be $35.5 \mathrm{ppb}$. From this result, the methane mixing ratios of the five DF standards were corrected by addition of $35.5 \mathrm{ppb}$ to the "assigned" mixing ratios. They are listed in Table 1. These "corrected" mixing ratios of the five DF standards were "tentative" values, because the correction is still quite rough. However, the "corrected" mixing ratios are thought to be closer to the absolute values than the "assigned" mixing ratios. Thus, the "corrected" mixing ratios of the methane calibration scale ("corrected" DF/NSC scale) used to determine the methane mixing ratio in the air of NOAA/CMDL cylinder (FF30507).

\subsection{Stability of standards}

The quality of atmospheric methane measurements depends on the stability of methane in te cylinders used as reference gases. To evaluate the long-term stability of our gravimetric standards, we continue to compare them with each other as to thier methane content. Fig.4 presents the history of the FID area response ratios among gravimetric standards of DJ, CPA and DF families. The area response ratios of the three standard families showed a small variation almost equal to our analytical precision over the course of 1-3 years. This result indicated that the methane level was stable in the high-pressure aluminum cylinders used for gravimetric standards from NSC. The stability of methane content in NSC standard cylinders was also reported by Aoki and Kawaguchi (1990) and Aoki et al.(1992).

Several steel cylinders filled with methane- 

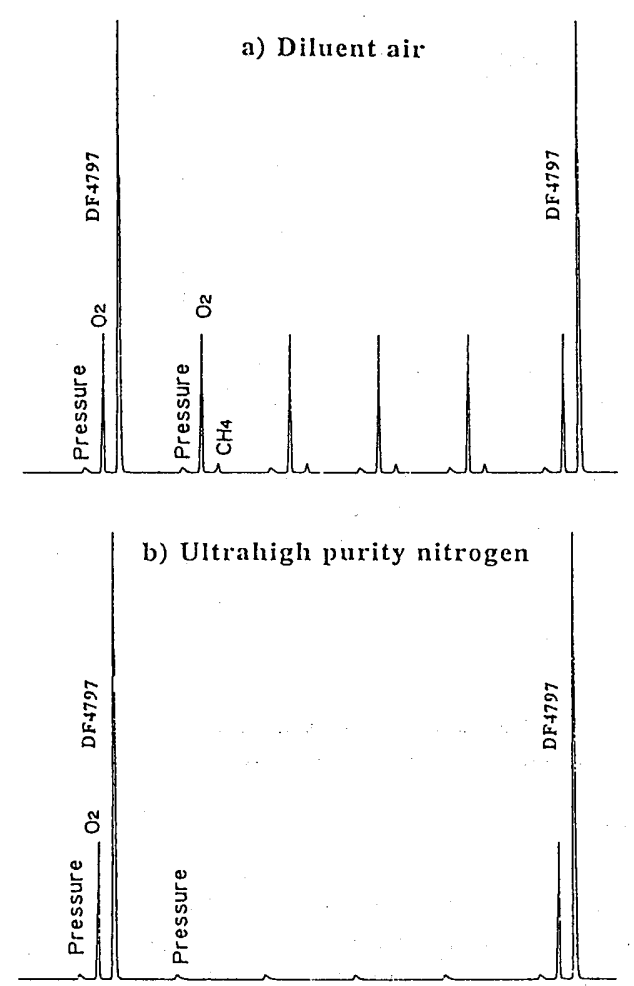

Fig. 3 Gas chromatograms of the diluent air (CPB00617) used for preparation of the DF standard family (a) and ultrahigh purity nitrogen (b). Gravimetric standard (DF4797) was injected before and after 4 analyses of the diluent air and ultrahigh purity nitrogen for comparison. in-aị have been used as secondary standards for methane measurements in atmospheric samples. We continued to compare the FID methane responses of all secondary standards to that of cylinder 2MK23241. The cylinder 2MK23241 was first used as a reference standard in our laboratory. Fig. 5 shows the history of the methane response of four secondary cylinders ratioed to that of cylinder 2MK23241. The area response ratios of all cylinders showed small variation with a standard deviation over the course of 1-5 years. Within our analytical precision, no drift in methane mixing ratio was found in the other steel cylinders, where the methane mixing ratio ranged from 1.5 to $2.2 \mathrm{ppm}$. These results indicated that methane was stable in the steel cylinders with brass fittings used for secondary standards from both NSC and TIC. As a result of stability of standards, we could confirm that our analytical system maintained good precision for at leat five years. Although all of our standard cylinders were stable with respect to methane, it is necessary to evaluate the long-term stability and to continue comparisons between the various standard gases.

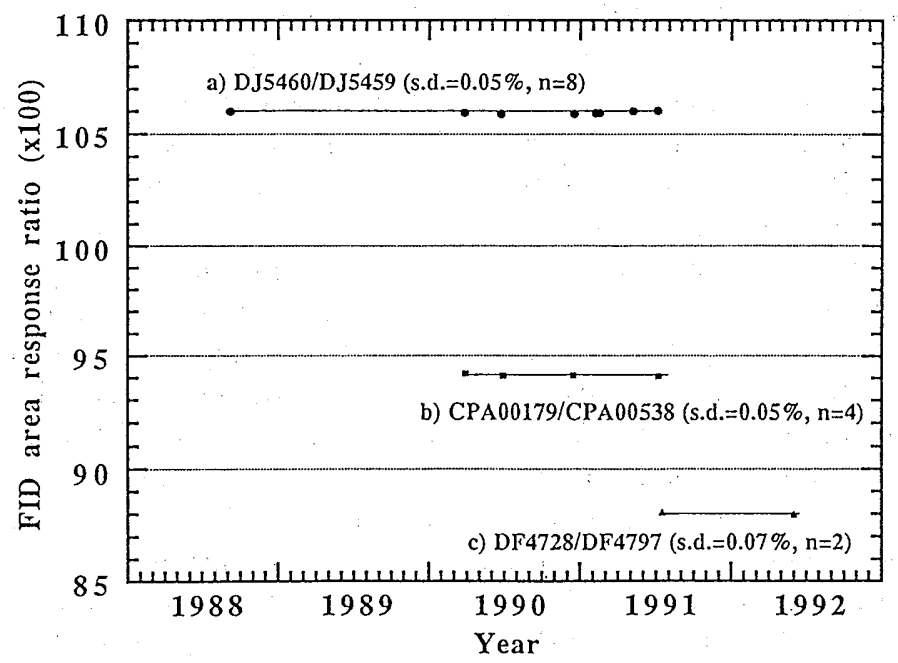

Fig. 4 Comparison of the FID area response ratios of DJ5460 to DJ5459(a), CPA00179 to CPA00538 (b) and DF4728 to DF4797 (c). Each point represents the mean of at least 5 area response ratios and its standard deviation is contained within each point. The solid lines represent the mean value of each data set. 


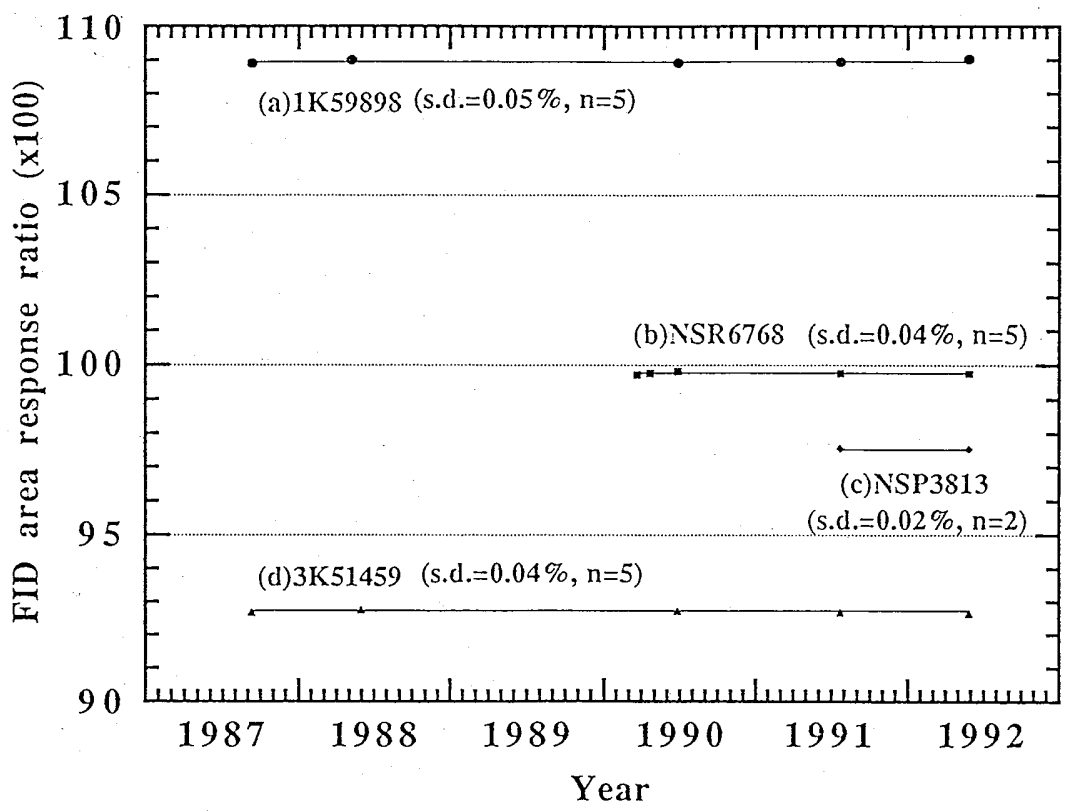

Fig. 5 Comparison of the FID area response ratios of secondary standards 1 K59898 (a), NSR6768 (b), NSP3813 (c) and 3K51459 (d) to that of secondary standard 2MK23241. Each point represents the mean of at least 5 area response ratios and its standard deviation is contained within each point. The solid lines represent the mean value of each data set.

\subsection{Methane mixing ratio of cylinder FF30507}

Cylinder FF30507 from NOAA/CMDL was an acculife treated, $5.9-\mathrm{L}$ aluminum cylinder made by Luxfer. This cylinder was filled with dry natural air from Niwot Ridge, Colorado, USA on 24 May, 1988. Before our analysis, the pressure of this cylinder was about 1100 psig, and it decreased by about 50 psig during the intercalibration experiment.

Five gravimetric standards from the DF family were used to determine the methane mixing ratio in the air of cylinder FF30507. As described above, the "corrected" methane mixing ratios were used as our calibration scale ("corrected" DF/NSC scale) in this experiment. These standard gases were first analyzed 5 times to test our GC/FID condition (chromatographic separation, linearity of GC response and analytical reproducibility). After that, 70 measurements for cylinder FF30507 were carried out from May 27 to June 3, 1992.
Prior to each measurement of FF30507, five standard gases of the DF family were analyzed. A straight line was fitted to the area response, and the mixing ratio for cylinder FF30507 was determined from this fit in this study. Each measurement was obtained by 5 repetitive analyses. The analytical reproducibilities for 5 analyses during this period were almost the same as those in Fig.1.

Fig. 6 shows the plot of mean area response versus methane mixing ratio for 75 measurements of the five DF standards over the course of May 27-June 3, 1992. The mean values of the five standards from the DF family were highly linearly correlated ( $\mathrm{r}=0.999994)$, indicating the linearity of our instrument. The analysis in each run also showed a high correlation coefficient of more than 0.9999 .

On the basis of these analyses, the methane mixing ratio of each standard gas was calculated using the best least squares fit of the other four standard gas measurements to eval- 


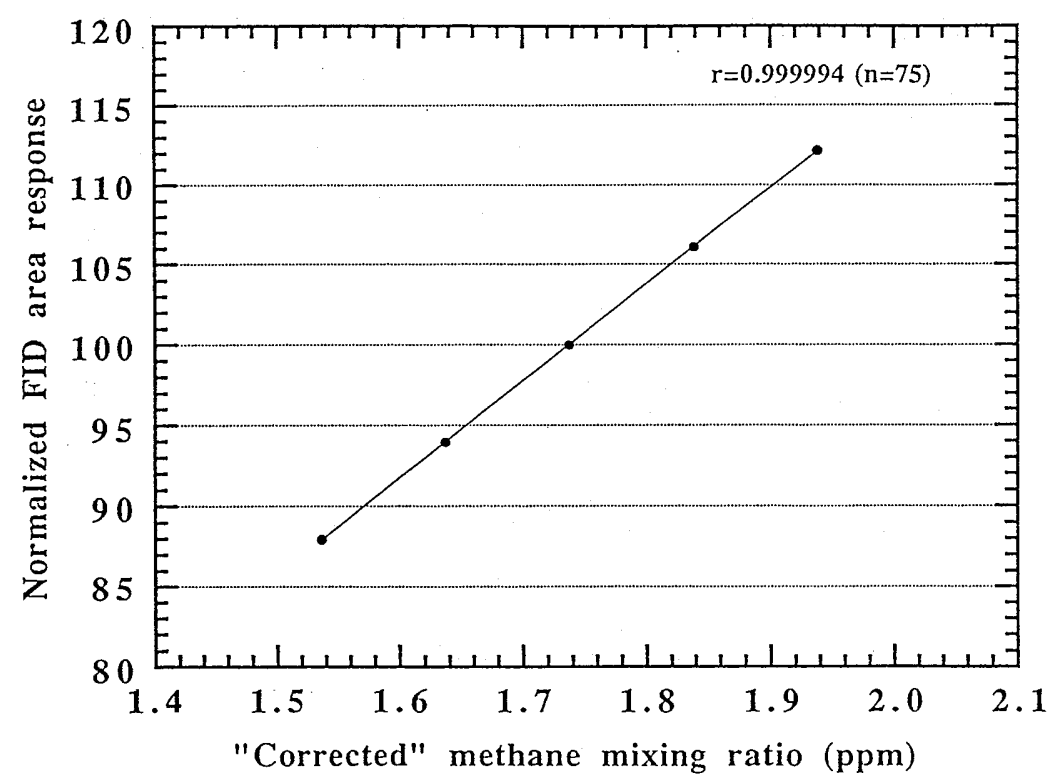

Fig. 6 Linearity of the GC/FID analysis system used in the intercalibration experiment during May 27-June 3, 1992. Five gravimetric standards of the DF family were used as the "corrected" methane mixing ratios. Each point represents the mean of 75 measurements and its standard deviation is contained within each point. The least squares line had a correlation coefficient $(r)$ of 0.999994 .

uate the internal consistency of five standards (Table 3). The calculated and "corrected" values agree with each other, indicating no abrupt change in the methane mixing ratios of the standards during this experiment. Thus, we were confident that our linear GC/FID analysis was valid for determining the methane mixing ratio of the air in cylinder FF30507.

Fig.7 shows the frequency distribution of the methane mixing ratios for FF30507 determined using five gravimetric standards of DF family. The total number of 70 measurements showed a mean mixing ratio of $1.7545 \mathrm{ppm}$ with 1 standard deviation ( 1 s.d.) of $0.0015 \mathrm{ppm}$. Of the 70 measurements, 27 were performed after drying the air through a stainless steel tube kept at $-80^{\circ} \mathrm{C}$ to check the water content. The analyses with drying showed a mean mixing ratio of $1.7545 \mathrm{ppm}(1$ s.d. $=0.0016 \mathrm{ppm})$, while the mean mixing ratio of 43 measurements without drying was $1.7546 \mathrm{ppm}$ ( 1 s.d $=0.0015$ $\mathrm{ppm})$. This excellent agreement strongly indicated no effect due to water vapor on the measured methane mixing ratio.
Table 3 Comparison between "corrected" $\mathrm{CH}_{4}$ mixing ratio and calculated $\mathrm{CH}_{4}$ mixing ratio using measurements of DF standard family during May 27-June 3, 1992.

\begin{tabular}{lccc}
\hline $\begin{array}{l}\text { Cylinder } \\
\text { number }\end{array}$ & $\begin{array}{c}\text { "Assigned" } \\
\text { mixing ratio } \\
\text { (ppm) } \\
\text { (A) }\end{array}$ & $\begin{array}{c}\text { CalculatedCH} \\
\text { mixing ratio* } \\
\text { (ppm) }\end{array}$ & $\begin{array}{l}\text { Percent } \\
\text { residual }\end{array}$ \\
& (B) & \\
\hline $\mathrm{DF} 4728$ & 1.5365 & 1.5380 & -0.098 \\
$\mathrm{DF} 4727$ & 1.6371 & 1.6366 & +0.031 \\
$\mathrm{DF} 4797$ & 1.7373 & 1.7366 & +0.040 \\
$\mathrm{DF} 4723$ & 1.8386 & 1.8382 & +0.022 \\
$\mathrm{DF} 4737$ & 1.9383 & 1.9398 & -0.077 \\
\hline
\end{tabular}

${ }^{*}$ Calculated $\mathrm{CH}_{4}$ mixing ratio was calculated from each measured area response using the best least squares fit to other four standard measurements. $\S$ Percent residual is defined as $[(\mathrm{A}-\mathrm{B}) / \mathrm{A}] \times 100$ and represents percentage deviation of the calculated $\mathrm{CH}_{4}$ mixing ratio to the "corrected" $\mathrm{CH}_{4}$ mixing ratio. 


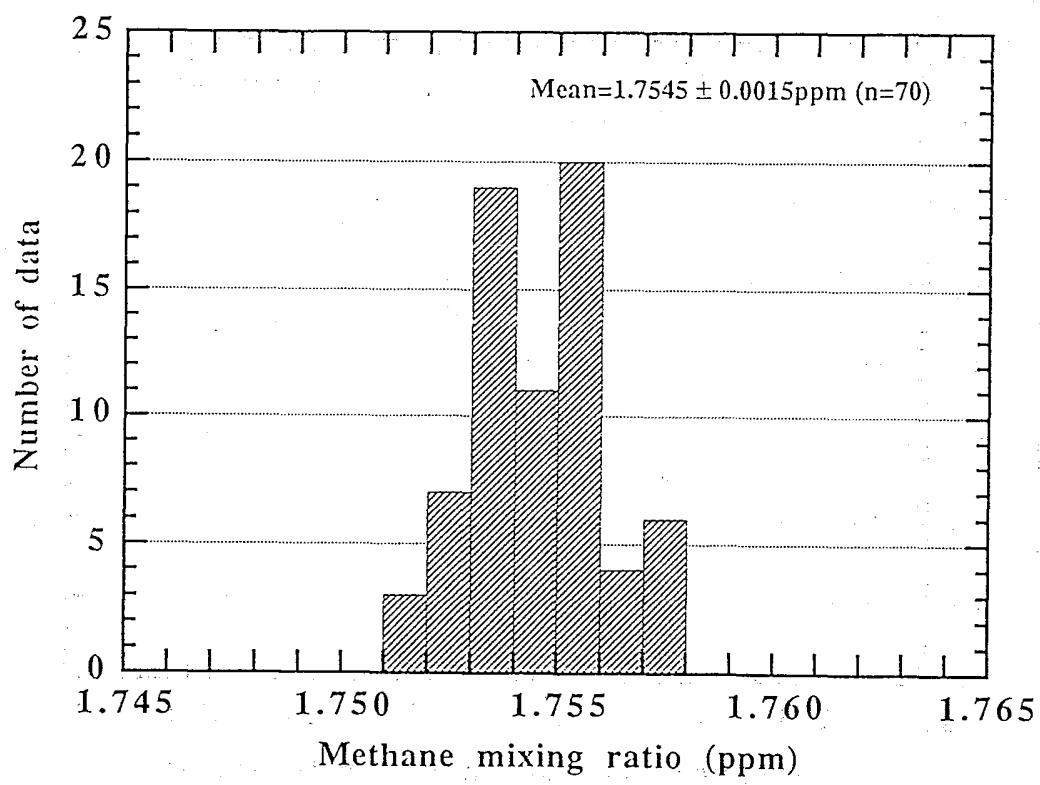

Fig. 7 Frequency distribution of methane mixing ratios in air of cylinder FF30507 determined 70 times using the five DF standards. The methane mixing ratio was calculated based on the "corrected" mixing ratio of the DF standard family (the "corrected" DF/NSC scale). The mean of 70 measurements is $1.7545 \pm 0.0015$ ppm( 1 s.d.). Details of the analysis and calculation procedure are given in the text.

\subsection{Comparison to NOAA/CMDL standard scale}

Various calibration scales are currently used by researchers measuring atmospheric methane (Rasmussen and Khalil, 1981; Blake and Rowland, 1986; Steele et al., 1987; Scheel et al., 1990; Aoki et al, 1992). Their scales were compared with a methane-in-air Standard Reference Material (SRM) prepared by NIST (the U.S. National Bureau of Standards and Technology, formerly the National Bureau of Standards (NBS) Washington D.C.). The disadvantage of the NIST standards is that they have concentrations of about $0.98 \mathrm{ppm}$ (SRM 1658a), 3.9ppm (SRM 1660a) and 9.5ppm (SRM 1659); which are largely different from atmospheric methane levels in the troposphere. There are no other reference standards which are available to insure the difference of calibration scale between our laboratory and others. Thus, this intercalibration experiment is essential to compare our atmospheric measurements with others.

The NOAA/CMDL calibration history for the cylinder FF30507 was reported as follows. Measurements of cylinder FF30507 were made on two different, but similar, automated gas chromatographs with flame ionization detectors. Each aliquot of a sample was bracketed by aliquots of a single standard gas. Mixing ratios were determined from peak heights. The measurements were referenced against two different primary standards consisting of dry natural air from Niwot Ridge, CO contained in acculife treated, aluminum cylinders. The NOAA/CMDL reference scale is propagated to each primary standard from a single cylinder of natural air purchased from Biospherics at the start of the methane measurement program by intense calibration. The scale is propagated to each new primary with an accuracy of \pm 0.2 ppb.

The mean value the NOAA/CMDl assigned to cylinder FF30507 was reported to be $1731.5 \pm 1.1 \mathrm{ppb}(1 \sigma)$. (Table 4 ). This mixing ratio was smaller by $23.0 \mathrm{ppb}$ than that determined by our laboratory using the "corrected" $\mathrm{DF} / \mathrm{NSC}$ standard scale. The difference 
between the two laboratories is thought to be due to several uncertainties of both calibration scales against an absolute scale. The difference of $23.0 \mathrm{ppb}$ is significant for comparison of atmospheric methane data from both laboratories since the magnitude of this difference is greater than the precision of GC/FID analysis. Thus, this experiment indicates that the offset of $23.0 \mathrm{ppb}$ between the two calibration scales will need to be taken into account when methane data from both laboratories are compared or be simultaneously used in future.

Table 4 Methane mixing ratio assigned to the air in cylinder FF30507 referenced against the NOAA/ CMDL scale and against two scales used in MRI/ GRL.

\begin{tabular}{|c|c|}
\hline Methane standard scale & $\begin{array}{l}\text { Methane mixning ratio } \\
\qquad(\mathrm{ppb})\end{array}$ \\
\hline NOAA/CMDL scale & $1731.5 \pm 1.1$ \\
\hline \multicolumn{2}{|l|}{ MRI/GRL scale } \\
\hline "Corrected"DF/NSC & scale $1754.5 \pm 1.5$ \\
\hline DJ/NSC scale & $1733.2 \pm 1.7$ \\
\hline
\end{tabular}

The "corrected" DF/NSC scale was not used for the atmospheric methane measurements previously reported by our laboratory (Inoue et al., 1991; Matsueda et al., 1992), because the DF standard gases based on the "corrected" DF/NSC scale were not obtained previously. The methane data published by Inoue et al. (1991) and Matsueda et al. (1992) were calibrated using the DJ standard gases, listed in Table 1. As shown in Fig. 2, the difference of calibration lines between the DF and DJ standard families were quantitatively determined. According to this difference, methane mixing ratios of cylinder FF30507 based upon the DJ/NSC reference scale were calculated and listed in Table 4. Thus, methane mixing ratios based upon the DJ/NSC scale (Inoue et al., 1991; Matsueda et al., 1992) are higher by only $1.7 \mathrm{ppb}$ than those determined in use at NOAA/CMDL scale.

Acknowledgements : - We thank Dr. Edward J. Dlugokencky of NOAA/CMDL for sending the cylinder to MRI/GRL as well as for reading this manuscript. This work was partly supported by The Special Coordination Fund for Promoting Science and Technology in Japan.

\section{References}

Aoki, S. and S. Kawaguchi, 1990: Development of high quality system for continuous measurement of atmospheric methane concentration (in Japanese). Antarctic Record, 34, 263-278.

Aoki, S., T. Nakazawa, S. Murayama and S. Kawaguchi, 1992: Measurements of atmospheric methane at the Japanese Antarctic Station, Syowa. Tellus, 44B, 273-281.

Blake, D. R. and F. S. Rowland, 1988: Continuing worldwide increase in tropospheric methane, 1978 to 1987. Science, 239, 1129-1131.

Fraser, P. J., P. Hyson, R. A. Rasmussen, A. J. Crawford and M. A. K. Khalil, 1986: Methane, carbon monoxide and methylchloroform in the Southern Hemisphere. J. Atmos. Chem., 4, 3-42.

Inoue, H. Y., H. Matsueda, T. Midorikawa and Y. Sugimura, 1991: Atmospheric $\mathrm{CO}_{2}$ and $\mathrm{CH}_{4}$ observed during the flight of International Strato/Tropospheric Air Chemistry I. Papers in Meteorology and Geophysics, 42, 3-10.

Khalil, M. A. K. and R. A. Rasmussen, 1986: Interannual variability of atmospheric methane: possible effects of the El Niño-Southern Oscillation. Science, 232, 56-58.

Matsueda, H., H. Inoue and Y. Sugimura, 1992: Atmospheric methane over the western Pacific and the Antarctic Ocean from 1978 to 1986. Geochem. J., 26, 21-28.

Matsueda, H., H. Inoue and M. Ishii, 1993: Latitudinal distributions of methane in the upper troposphere and marine boundary air over the Pacific in 1990. Geophys. Res. Letters, 20, 695-698.

Rasmussen, R. A. and M. A. K. Khalil, 1981: Atmospheric methane (CH4): Trends and Seasonal cycles. J. Geophys. Res., 86, 9826-9832.

Scheel, H. E., E. -G. Brunke and W. Seiler, 1990: Trace gas measurements at the monitoring station Cape Point, South Africa, between 1978 and 1988. J. Atmos. Chem., 11, 197-210.

Steele, L. P., P. J. Fraser, R. A. Rasmussen, M. A. K. Khalil, T. J. Conway, A. J. Crawford, R. H. Gammon, K. A. Masarie and K. W. Thoning, 1987: The global distribution of methane in the troposphere. J. Atmos. Chem., 5, 125-171.

Steele, L. P., E. J. Dlugokencky, P. M. Lang, P. P. Tans, R. C. Martin and K. A. Masarie, 1992: Slowing down of the global accumulation of 


\section{NOAA/CMDLとMRI/GRLとの間のメタン標準ガス・スケールの相互比較実験}

\section{松枝秀和}

米国大気海洋局 (NOAA/CMDL) と気象研究所(MRI/GRL)で使用しているメタン標準ガスのスケールの違 いを明らかにするため相互比較実験を実施した。相互検定用ボンべに充媜された空気のメタン混合比をMRI/ GRLのスケールを基準として測定した結果, $1.7545 \pm 0.0015 \mathrm{ppm}$ の值が得られた。これは, NOAA/CMDLによっ て值付けされた混合比に比べ23.0ppb高いことがわかった。この実験を通じて，MRI/GRLのメタンのデータが NOAA/CMDLと同じスケールで比較することが可能になった。 